\title{
Giovanni Inzerillo
}

Università di Palermo

\section{"IL BARBARO È VINTO". \\ D'ANNUNZIO CONTRA WAGNER. DAL SINFONISMO TEDESCO ALLA MUSICA DEL SILENZIO NEI ROMANZI DI GABRIELE D'ANNUNZIO}

Soltanto alla musica è oggi dato esprimere $i$ sogni che nascono nelle profondità della malinconia moderna, $i$ pensieri indefiniti, $i$ desideri senza limiti, le ansie senza causa, le disperazioni inconsolabili, tutti i turbamenti più oscuri e più angosciosi.

(G. D’Annunzio)

Se resta ineccepibile il dato, indubbiamente significativo, che alla Coscienza sveviana sia da ascriversi la modernità del romanzo italiano novecentesco con l'avvento della psicoanalisi e lo scardinamento del tempo in chiave proustiana (il tempo della narrazione è sempre il presente della coscienza e non la cronologia dei fatti descritti), altrettanto ineccepibile è il fatto che, già alla fine dell'Ottocento, D’Annunzio prova a sperimentare una forma di prosa moderna prima di Svevo e con altri esiti. D'altronde, è nell'intento dello stesso autore, come si legge nella dedica a Michetti ad apertura del Trionfo, la precisa intenzione di creare un «ideal libro di prosa moderno»:

Avevamo più volte insieme ragionato di un ideal libro di prosa moderno che essendo vario di suoni e di ritmi come un poema, riunendo nel suo stile le più 
diverse varietà della parola scritta - armonizzasse tutte le varietà del conoscimento e tutte le varietà del mistero [...] v'è, sopra tutto, il proposito di fare opera di bellezza e di poesia, prosa plastica e sinfonica, ricca di immagini e di musiche. [...] hanno elementi musicali così varii e così efficaci da poter gareggiare con la grande orchestra wagneriana nel suggerire ciò che soltanto la Musica può suggerire all'anima moderna'.

L'interesse per la musica dimostrato da D'Annunzio in tutti i suoi romanzi non può essere ricondotto ad una semplice questione di gusto o sensibilità e, come afferma la Silvana Cellucci Marcone nel suo D'Annunzio e la musica,: «la scarsa attenzione che gli studiosi hanno dedicato a questo argomento si può giustificare solo con la considerazione che la musicalità che è nell'opera del Nostro è stata posta in relazione con certi aspetti della sensibilità decadente, piuttosto che con precisi rapporti con maestri e compositori contemporanei al poeta» ${ }^{2}$.

Fino agli anni Settanta, infatti, la critica aveva riservato poco spazio allo studio della musicalità nell'opera dello scrittore abruzzese. Ad una netta inversione di tendenza si assiste solo a partire dall'ultimo ventennio del secolo scorso con gli studi condotti da Adriana Guarnieri Corazzol, da Paola Sorge e da Rubens Tedeschi.

Il libro della Marcone, sebbene ormai superato dagli studi più recenti, continua ad essere, ancora oggi, di grande attualità laddove riporta lettere inedite e aspetti altrettanto poco conosciuti delle collaborazioni tra il poeta e numerosi musicisti del suo tempo come Debussy, Tosti, Franchetti, Pizzetti, Mascagni e Zaldonai.

Le vicende biografiche dannunziane e gli scritti autocelebrativi permettono di comprendere come la passione per la musica, presto tramutatasi in musicalità letteraria vera e propria, ha preso corpo proprio a partire da una esperienza di vita, è maturata in relazione a un giovanile impegno fallito, causa l'inadeguatezza per un'arte difficile e poco accessibile. Educato sin da bambino allo studio del pianoforte sotto la guida del Maestro Odoardo Chiti e intrapresi gli studi di canto in età adolescenziale, D'Annunzio non superò mai le difficoltà tecniche per diventare un bravo musicista. Lo scrittore e drammaturgo francese Romain Rolland, legato a D'Annunzio da intima amicizia, riferisce lo stato d'animo del Vate sconfortato dalla propria inadeguatezza: «Gli suonai al pianoforte musiche di ogni

\footnotetext{
1 D’ANNUNZIO G. (1995), Trionfo della morte, Mondadori, Milano, pp. 3-8.

2 CELlUCCI MARCONE S. (1972), D’Annunzio e la musica, Japadre, L'Aquila 1972, p. 5.
} 
epoca [...] l'Adagio dell'ultimo Quartetto di Beethoven lo portò alle lacrime [...] la sera, quando era solo, provava a tentoni a improvvisare» ${ }^{3}$.

Leggendo gli espistolari non deve stupire, allora, il tono autenticamente reverenziale con cui il poeta dialoga con i grandi musicisti del suo tempo come, ad esempio, Debussy. Il "Sommo Vate", rivolgendosi al Maestro francese con il tono rispettoso e prudente di un fanciullo, attraverso l'utilizzo di costruite ed eleganti formule pleonastiche e il ricorso ad affettuosi epiteti, attua una celata ma insistente captatio benevolentiae:

Vi conoscevo e vi amavo già allora. [...] Allora, come oggi, soffrivo di non potere scrivere la musica delle mie tragedie. [...] Scrivetemi soltanto una parola e sarò da voi. Avrò almeno la gioia di dirvi tutta la mia riconoscenza per i bei pensieri che qualche volta avete cullato e nutrito nell' anima mia senza pace. [...] L'idea di lavorare con voi, anzi, mi dà in anticipo una sorta di febbre. [...] Non ho mai sentito con forza maggiore la profonda novità della vostra arte. Arrivederci. Ora, e lo sapete, vi amo quanto vi ammiro ${ }^{4}$.

Il dato autobiografico si traduce, così, in invenzione letteraria. La musica entra prepotentemente nelle opere di D'Annunzio passando proprio per una esperienza di vita, traendo origine e motivazione dal tentativo, subito sfociato in amaro fallimento, di diventare egli stesso un musicista. Paradossalmente, però, questa ossessione musicale, che per D'Annunzio avrebbe dovuto far risaltare la sua abilità di scrittore procurandogli lodi e prestigio, ha sancito, in un certo qual modo, la sua condanna. I giudizi espressi dalla critica recente sono tutt'altro che lusinghieri nel delineare le madornali inesattezze e le debolezze di uno scrittore tutt'altro che vate, arrogante, mistificatore e persino plagiatore. Scrive Rubens Tedeschi:

Dei rapporti tra D'Annunzio e la musica sappiamo tutto, e anche qualcosa di più, grazie alla costante mistificazione autobiografica diretta a trasformare il quotidiano in sublime. Passando dalle ginocchia di Bach al feretro di Wagner, il vate si auto-

${ }^{3}$ A tal proposito si legga: tosi G. (1963), D’Annunzio visto da Romain Rolland, La Nuova Italia, Firenze. Per altre informazioni sulla vita del poeta e specie sui suoi rapporti col teatro si consiglia: ANTONGINI T. (1938), Vita segreta di Gabriele D’Annunzio, Mondadori, Milano.

4 D'ANNUNZIO G. - DEBUSSY C. (1948), Claude Debussy et Gabriele D'Annunzio: correspondence inédite, Denoel, Paris (trad. it. Mon cher ami, Epistolario 1910-1917, Passigli, Firenze 1993), pp. 23, 25, 33. A tal proposito si consiglia: STEFANIA FILIPPI, Il carteggio fra D'Annunzio e Debussy, in: "Bollettino '900", rivista ellettronica di letteratura contemporanea, a cura dell’Università di Bologna, dicembre 2001 (http://www2.unibo.it/boll900/numeri/2001-ii/W-bol/ Filippi/). 
consacra musico: maestro di polifonia, esperto nell'intrecciare voci nel contrappunto fiammingo appreso sui testi di Josquin Desprès e di Giovanni Pierluigi da Palestrina. [...] Altrove si proclama «allevato sulle ginocchia della musica» 0 , più specificatamente, «sulle ginocchia congiunte di Bach». [...] L'aulica mistificazione serve a mobilitare una realtà più modesta. Gli studi musicali non sono andati oltre i primi rudimenti. [...] Siamo, non occorre sottolinearlo, nel regno della fantasia che nutre gran parte dell'autobiografia dannunziana ${ }^{5}$.

Se è vero che l'ostentata conoscenza musicale nelle opere dello scrittore ha molto di artefatto (Tedeschi parla correttamente di «musica come aspirazione», mentre la Corazzol utilizza i termini di «riesumazione»e «feuilleton» ${ }^{6}$ ), non va tuttavia sottovalutata l'originalità e l'innata predisposizione verso una scrittura di tipo teatrale fortemente intrisa, soprattutto nella prosa, di un marcato simbolismo musicale.

Sebbene nel Piacere (1889) D'Annunzio dia ampia prova, nonostante la vibrante passione che anima la scrittura, di una scarsa competenza musicale, ad ogni modo nel romanzo traspare, nella resa stilistica ed espressiva, una musicalità della parola capace di esprimere raffinate emozioni e sensibilità. I riferimenti, per citare solo alcuni esempi, alla celebre sonata di Beethoven op. 27 n. 2 vagheggiano un sentimento erotico e sensuale; i commenti, solo fintamente più dettagliati, al Quartetto in do minore di Brahms smascherano, è vero, una vaghezza da dilettante, specie negli accostamenti con Bach e Beethoven creati dallo scrittore, ma sono comunque capaci di trasferire efficacemente in immagini le emozioni suscitate dall'ascolto:

A poco a poco la musica grave e soave prendeva tutti que' leggeri spiriti ne' suoi cerchi, come un gorgo tardo ma profondo. - Beethoven - disse Elena, con un accento quasi religioso, arrestandosi e sciogliendo il suo braccio da quello di Andrea. Ella così rimase ad ascoltare, si metteva un guanto, con estrema lentezza. In quell'attitudine l'arco delle sue reni appariva più svelto; tutta la sua figura, continuata dallo strascico, appariva più alta ed eretta; l'ombra della pianta velava e quasi direi spiritualizzava il pallore della carne. Andrea la guardò. E le vesti, per

5 TEDESCHI R. (1988), D’Annunzio e la musica, La Nuova Italia, Firenze, pp. 7-9.

${ }^{6}$ Si vedano rispettivamente: TEDESCHI R. (1988), D’Annunzi... e guarnieri corazzol A. (1990), Sensualità senza carne. La musica nella vita e nell'opera di D’Annunzio, Il Mulino, Bologna. 
lui, si confusero con la persona. «Ella sarà mia» pensava, con una specie d'ebrietà perché la musica gli aumentava l'eccitamento ${ }^{7}$.

(Brahms) Il primo tempo esprimeva un lottar cupo e virile, pieno di vigore. La Romanza esprimeva un ricordarsi desioso ma triste, e quindi un sollevarsi lento, incerto, debole, verso un'alba assai lontana. Una chiara frase melodica si svolgeva con profonde modulazioni. Era un sentimento assai diverso da quello che animava l'Adagio di Bach; era più umano, terreno, più elegiaco. Passava in quella musica un soffio di Ludovico Beethoven ${ }^{8}$.

Per quanto la trasposizione delle note in parole raramente regga a una analisi attenta e le osservazioni più squisitamente musicali siano poco pertinenti - ragion per cui è pressoché impossibile, se non previa indicazione autoriale, rintracciare i brani musicali citati - , come efficacemente ribadito dalla Corazzol, nei romanzi dannunziani si realizza: «quella concezione simbolistica della musica» che altro non è se non «un vero e proprio esercizio di stile» ${ }^{9}$.

Specificatamente agli esordi letterari di D'Annunzio, il linguaggio musicale viene tradotto soprattutto in termini sentimentali ed esprime un gusto da salotto raffinato ma poco colto. Non stupisce allora che delle trendadue sonate composte da Beethoven (escludendo le tre «Sonate all'Elettore») venga citata la più celebre, ma anche la più volgarmente discussa, Al chiaro di luna, di cui la triste dolcezza del primo movimento Adagio sostenuto è stata, da sempre, abusata dai più melliflui sentimentalismi di appassionati, non esperti, di musica e ha catturato così anche gli animi sensibili delle giovani fanciulle nei salotti dell'epoca ${ }^{10}$.

Sebbene per la maggior parte della critica in tutti i romanzi di D'Annunzio la musica assuma sempre un ruolo di subordinarietà rispetto alla narrazione, questa risulta più che mai necessaria per dare enfasi e sensibilità ad una scrittura di per sé assai poco emotiva. Efficace, in tal senso, è il concetto di «sensualità senza carne» coniato dalla Corazzol che, volendo andare oltre il gusto e le intenzioni narrative dello scrittore, ben sottolinea l'emotività di D'Annunzio sensibile alla musica ma non musicista, la «sensibilità» musicale priva della sostanza teorica e tecnica vera e propria, della materia che la plasma, della «carne» per l'appunto.

\footnotetext{
7 D’ANNUNZIO G. (1989), Il piacere..., p. 30.

8 Ibidem, p. 137.

9 GUARNIERI CORAZZOL A. (1990)., Sensualità senza carne..., p. 123.

10 CFR. TEDESCHI R. (1988), D’Annunzio..., p. 14.
} 
La musica, pertanto, esprime i più svariati contenuti, erotici e sensuali in questa prima fase, fuori da qualsiasi concettualismo tecnico o critico $^{11}$.

Ancora più complessi risultano essere i romanzi successivi più marcatamente musicali. In essi i richiami alle opere, e alla figura in prima persona, di Wagner hanno la loro dovuta rilevanza. Il grande musicista tedesco cattura le attenzioni e la stima di D'Annunzio che, vantando un profondo legame di intelletto e di sensibilità con il Maestro, in un articolo apparso in tre puntate tra il luglio e l'agosto del 1893 sul giornale «La Tribuna», non esita a scagliarsi contro il Nietzsche spietato e polemico del celebre Caso Wagner e del Nietzsche contra Wagner composti entrambi nel 1888 .

Le feroci accuse e le aspre invettive reiterate dal filosofo ai danni di Wagner, frutto talvolta di arroganza intellettuale e spesso fondate su edifici argomentativi poco complessi, hanno tuttavia le loro precise e solide giustificazioni.

Già nella Prefazione al Caso Wagner, Nietzsche vuole mettere in luce le ragioni della sua critica e le concrete motivazioni. Non è una banale questione di gusto musicale a differenziare così enormemente artisti contemporanei e per giunta compatrioti. Il filosofo infatti pone subito l'accento sulle due questioni che gli stanno maggiormente a cuore: la modernità e la décadence.

Il «dannoso» Wagner, a detta di Nietzsche, incarna per eccellenza la modernità («Wagner è l'artista moderno par excellence») ma quella modernità nella quale egli stesso, seppur contemporaneo, non osa e non vuole riconoscersi. Essa porta con sé uno spirito decadente, bigotto e moralisticamente cristiano, che ha come sintomi «la vita impoverita», «la volontà della fine» e «la grande stanchezza». Nietzsche preferisce piuttosto osservare il suo tempo con uno sguardo lucido ma freddo, disincantato ed estraneo:

Che cosa esige da sé un filosofo come prima e ultima cosa? Di superare in sé il proprio tempo, di diventare «senza tempo». Con che cosa allora deve sostenere il suo cimento più duro? Proprio con quello per cui è figlio del suo tempo. Orbene, io sono altrettanto di Wagner figlio di questo tempo, voglio dire un décadent; solo

${ }^{11}$ Cfr. GUARNIERI CORAZZOL A. (1990), Sensualità senza....: "In quest'ermeneutica visionaria ormai di massa ciò che importa non è il linguaggio della musica ma la sua traduzione in termini sentimentali, il suo effetto sulla fantasia o sui nervi dei protagonisti del romanzo: i suoni sono, romanticamente, suscitatori di immagini oppure, naturalisticamente, veicoli di sensazioni. [...] I brani ascoltati da D'Annunzio hanno come unico senso quello di veicolare l'ineffabile sentimentale. [...] Nel contesto della situazione narrativa la musica è estensione dell'erotismo, 'volo' che dalle cime dell'estasi spirituale prima o poi si concreta in immagine di piacere carnale. [...] Nelle pagine romanzesche di D'Annunzio il simbolo musicale si dichiara bensì purificazione e decantazione di tutti i sensi, ma esprime e figura la carne", pp. 129-130, 138-139, 142. 
che io l'ho capito, solo che io me ne sono difeso. Il filosofo in me se n'è difeso. Quello che mi ha occupato più a fondo è in realtà il problema della décadence. [...] Se ci si è fatto l'occhio per i sintomi della décadence, si capisce anche la morale - si capisce che cosa si nasconde sotto i suoi nomi e le sue formule di valore più sacri: la vita impoverita, la volontà della fine, la grande stanchezza. La morale nega la vita. [...] un profondo estraneamento, raffreddamento, disincanto per tutto quanto è attuale e conforme allo spirito del tempo. [...] La mia esperienza più grande fu una guarigione. Wagner fa parte semplicemente delle mie malattie. [...] Attraverso Wagner la modernità parla la sua lingua più intima: non nasconde né il suo bene né il suo male, ha disimparato ogni vergogna di sép ${ }^{12}$.

Sebbene quindi Wagner sia per Nietzsche una «malattia» («Wagner in genere un essere umano? Non è invece una malattia? Egli fa ammalare tutto ciò che tocca - ha fatto ammalare la musica $\left.{ }^{13} \gg\right)$, la sua indiscussa modernità è concepita, in negativo, come una drammatica «corruzione del gusto» e come una insanabile «contraddizione di valori». Wagner, il seduttore delle masse, nelle vesti di «commediante della musica» ha saputo come un tiranno catturare il grande entusiasmo popolare ${ }^{14}$. La passione, da Nietzsche definita la «ginnastica del brutto», sconvolge le masse facilmente suggestionabili.

I giudizi espressi dal filosofo non riguardano soltanto la decadenza del gusto ma si fanno più impietosi quando toccano questioni di stile musicale. Wagner, visto ancora oggi come artista della totalità e del pieno sinfonismo, da Nietzsche viene drasticamente ridimensionato e la sua musica viene ritenuta povera nello stile, scarna nella struttura, inautentica, artefatta e persino di poca sostanza («Wagner non sapeva creare dalla totalità»). È pur vero che le considerazioni squisitamente tecniche costituiscono una minima parte delle argomentazioni nietzscheane. Ciò che maggiormente preme al filosofo resta, per la quasi totalità

12 NIETZSCHE F. (2007), Il caso Wagner, in Scritti su Wagner, a cura di Sossio Giametta, Rizzoli, Milano, pp. 57-58. I corsivi sono nel testo.

13 Ibidem, p. 67.

${ }^{14}$ Cfr. ibidem, pp. 83-84, 89: "Ho spiegato qual è il posto di Wagner - non nella storia della musica. Che cosa significa egli tuttavia in questa? L'avvento del commediante della musica: un evento capitale che dà da pensare e che forse dà anche da temere. [...] Sorge così l'età aurea per il commediante - per lui e per tutto quanto è affine alla sua specie. Wagner marcia con tamburi e pifferi alla testa di tutti gli artisti della declamazione, della rappresentazione e del virtuosismo; ha convinto per primi i direttori d'orchestra, i macchinisti e i cantanti di teatro. [...] Il teatro è una forma di demolatria nelle cose del gusto, il teatro è un'insurrezione delle masse, un plebiscito contro il buon gusto...Appunto questo dimostra il caso Wagner: egli conquistò la moltitudine corruppe il gusto, corruppe perfino il nostro gusto per l'opera!'. I corsivi sono nel testo. 
dell'opera, quella finta moralità di cui Wagner si è fatto, attraverso i personaggi delle sue opere e i dialoghi da lui stesso composti, portavoce e imbonitore, quasi fosse un Cristo moderno («l'opera wagneriana è l'opera della redenzione»). Ed è proprio questa la maggiore ingenuità di Nietzsche: il volere a tutti i costi «applicare all' arte un criterio morale» (Sossio Giametta) $)^{15}$. Non è un caso che faccia da Epilogo una breve ma dettagliata riflessione non solo su questioni di estetica (l'estetica della décadence in opposizione all'estetica classica) ma, soprattutto, su questioni di etica (quella distinzione tra la morale dei valori cristiani e la morale dei signori da cui D'Annunzio prenderà le mosse ricavandone le sue argomentazioni a difesa di Wagner). Sebbene «la morale dei signori afferma altrettanto istintivamente che quella cristiana nega», queste sono «entrambe necessarie» perché il mondo moderno riassume in sé la contraddittorietà. Wagner, ed è questa la sua colpa per Nietzsche, ha piuttosto preferito la morale cristiana della rassegnazione e della pietà, della speranza e della serenità, esaltando come valori le fortune di una vita stanca e inattiva.

Cinque anni più tardi la pubblicazione degli scritti su Wagner, D’Annunzio non esita a replicare a Nietzsche in difesa del Maestro. Proprio in quell' anno lo scrittore abruzzese stava ultimando la stesura del Trionfo, apparso l'anno successivo (1894). Leggere le invettive del filosofo sembrava, in un certo qual modo, mettere in discussione lo stile allora intrapreso, tutto contaminato da una musicalità di chiara ispirazione wagneriana. Una risposta al testo di Nietzsche appariva dunque necessaria, quasi d'obbligo.

Eppure la conclusione della dedica al Michetti sembra contraddire il pensiero wagneriano di cui il romanzo vuole farsi rivisitazione:

Noi tendiamo l'orecchio alla voce del magnanimo Zarathustra, o Cenobiarca; e prepariamo nell'arte con sicura fede l'avvento dell'UEBERMENSCH, del Superuomo ${ }^{16}$.

Il pensiero filosofico nietzscheano, a cui D'Annunzio vuole estendere l'arte tout court, vuole essere la vera matrice della sua scrittura. Eppure, come ribadito da Paola Sorge, nello scrittore sembra ci sia ben poco di Nietzsche e della sua filosofia e «quell'etichetta nietzscheana serviva solo da sfida» ${ }^{17}$. Nietzsche infatti,

${ }^{15}$ Cfr. GIAMETTA S., Gli scritti su Wagner nell'opera di Nietzsche, in: Nietzsche F., op.cit., pp. 5-8.

16 D'ANNUNZIO G., Trionfo della morte, op.cit., p. 8. Il maiuscoletto è nel testo.

17 SORGE P. (1996), D’Annunzio tra Wagner e Nietzsche, in: GABRIELE D'ANNUNZIO, Il caso Wagner, a cura di Paola Sorge, Laterza, Bari, p. 14. Sui rapporti tra lo scrittore e Wagner, 
assai frequentemente abusato in relazione alle opere del Nostro, era preso spesso a modello non tanto per le sue teorie filosofiche, quanto come ideale di lotta antidemocratica e antipartitica. D'altronde, Andrea Sperelli, Giorgio Aurispa e Stelio Effrena, solo per citare alcuni tra i protagonisti più noti dei suoi romanzi, cosa sono se non degli inetti dalle chiare fattezze decadenti? Cosa c'è in loro di attivo, di superomistico? Scrive ancora la Sorge:

Quella del D'Annunzio nietzscheano è in realtà una etichetta semplificatoria, comoda per tutti, incluso il poeta stesso [...] Il «Barbaro enorme» non ha nulla da insegnargli. Ed è indubbiamente vero, dato che Gabriele D'Annunzio è esattamente il contrario degli ideali nietzscheani; artista decadente per eccellenza, sembra incarnare lui, e non Wagner, la malattia del secolo, quella décadence tanto aborrita dal filosofo. [...] Le dottrine del filosofo, lette superficialmente, non intaccarono affatto l'essenza della sua opera [...] Il superomismo che entra trionfalmente nella dedica a Michetti del Trionfo della morte e che sembra improntare da questo momento la sua opera, in realtà non ha nulla della forza provocatoria, dissacrante, rivoluzionaria del pensiero nietzscheano ${ }^{18}$.

Come si è già detto, furono tre gli interventi scritti da D'Annunzio in difesa di Wagner, poi pubblicati con il titolo, analogo a quello dell'opera nietzscheana, di Caso Wagner.

Nel primo del 23 luglio 1893 lo scrittore esalta le doti intellettuali di Nietzsche spiegandone alcune teorie filosofiche. Esordendo anche lui con una critica rivolta, senza mezzi termini, contro la bibliografia wagneriana allora in circolazione, è curioso notare (fatto quasi divertente alla luce delle scoperte recenti) come D'Annunzio tra i suoi bersagli prenda anche di mira lo scritto di Nerthal, Tristan et Yseult: la passion dans un Drame Wagnérien, da lui considerato «stupido» e «vacuo». Ingenuità madornale: parlar male proprio di un testo da cui, come si è già accennato, copia pedissequamente molti dei commenti al Tristano inseriti nel Trionfo ${ }^{19}$ !

sebbene analizzati sotto un'ottica di insistente ammirazione per D'Annunzio e di poco onesta indagine critica (fatto pure comprensibile per l'epoca della pubblicazione) si consiglia inoltre: DONATI PETTÉNI G. (1923), D’Annunzio e Wagner, Le Monnier, Firenze.

18 Ibidem, pp. 7-8, 16-17.

19 Ecco solo due della quasi totalità degli esempi riscontrabili: "Ecco apparire Tristano davanti alla furente Isotta, mentre dall'alto dell'albero, la vedetta riprendeva la sua canzone, su l'onda saliente dell'orchestra" (En haut du mat reprend la chanson appuyée cette fois et soutenue par le flot montant de l'orchestre, Nerthal); "Il filtro di morte non era se non un veleno d'amore che li 
Dopo aver presentato il filosofo tedesco come «uno dei più originali spiriti che siano comparsi in questa fine di secolo, ed uno dei più audaci», si concentra poi a spiegarne la teoria della morale (la morale dei nobili e la morale del gregge servile) e l'ambiguità dei concetti di Bene e Male. La morale del gregge servile si è imposta all'altra, causa anche il dominio intellettuale esercitato dal Cristianesimo sulle masse, e «l' ascetismo diffuse un velo di pallore e di tristezza su tutte le cose». Per D'Annunzio è chiaro, dunque, il perché Nietzsche odiasse Wagner: allo spirito rivoluzionario e vitalistico del filosofo si opponeva l'artista rassegnato e decadente. Si legge infatti:

La musica di Riccardo Wagner è la musica della democrazia socialista in contrapposto all' arte aristocratica, eroica o soggettiva. Rappresenta l'abdicazione dell'Io e l'emancipazione di tutte le forze vinte. Risponde alle tendenze dell'epoca. Le quali disconoscono il vero valore della personalità umana sommergendolo nel complesso della natura o della società. Il Nietzsche dunque - che, come abbiamo veduto, ha un ideale di vita ascendente - riconosce e combatte in Riccardo Wagner il tipo esemplare dell'artista decadente, riconosce ed abomina in lui tutte le debolezze e tutte le infermità del secolo ${ }^{20}$.

Il secondo intervento, apparso il 3 agosto del 1893, è interamente dedicato alla contraddittoria figura di Wagner. Sin da subito la sua natura viene presentata come «una delle più complicate, delle più inquiete, delle più mobili, delle più contraddittorie che questo secolo offra alla curiosità degli psicologi» ${ }^{2121}$. Analizzando la genesi e i contenuti di alcune opere wagneriane, D'Annunzio sembra paradossalmente confermare i giudizi espressi dal filosofo che pure aveva rapidamente passato in rassegna le opere da lui definite «della redenzione». Wagner esordisce con vigore ottimistico, scagliandosi contro la latinità e l'ipocrisia del Cristianesimo ed esaltando la sensualità e la passionalità dell' amore corporeo e non ascendente ${ }^{2222}$. Tale vitale ottimismo viene però in un secondo momento messo da parte e tale trasformazione è dovuta, per D'Annunzio, a un acuirsi della sensibilità (cita persino una lettera scritta a Liszt dove Wagner riferisce di essersi completamente immedesimato in Dante durante una lettura della Commedia).

penetrava d'un fuoco immortale" (Ce poison de mort est un poison d'amour qui leur met le feu dans les veines, Nerthal).

20 D'ANNUNZIO G., Il caso Wagner..., pp. 54-55. Il corsivo è nel testo.

21 Ibidem, p. 57.

${ }^{22}$ Cfr. ibidem, p. 60: «Egli intendeva, con questo, che l'amore dovesse essere l'affermazione della vita. Egli aspirava alla vita ascendente, alla forza, alla sanità, alla piena gioia». 
Mentre per Nietzsche tale radicale trasformazione della sensibilità wagneriana avveniva molto presto, già con la composizione del Sigfrido, per D'Annunzio fu il Parsifal a fare da vero e proprio spartiacque. Al Sigfrido, poema dell' ascesi, si contrappone così il Parsifal, poema della rinunzia. Nulla di nuovo rispetto alle teorie nietzscheane, quindi. Basta porre a confronto i giudizi espressi da entrambi:

Il Wagner dell'aspirazione alla salute, alla forza, alla gioia, alla giovinezza, a tutte le virtù della vita ascendente inchina verso le virtù contrarie, verso la morale negativa, verso l'Evangelo dell'umiltà, verso la Rinunzia ${ }^{23}$.

Wagner si arenò su un'opposta visione del mondo. Che cosa aveva egli messo in musica? L'ottimismo. Wagner se ne vergognò ${ }^{24}$.

Una vera e propria difesa a favore del musicista avviene solo nel terzo intervento del 9 agosto 1893. In esso D'Annunzio cita frequentemente Nietzsche e replica alle critiche di «istrionismo» e di assenza di drammaturgia musicale in riferimento alle opere di Wagner. Ecco un significativo esempio in cui lo scrittore, insieme ai riconosciuti meriti di alcuni dei giudizi espressi dal filosofo, ben a ragione ne biasima le madornali incoerenze del pensiero e della scrittura:

Qui è il grossolano errore o la vana ingiustizia. Per me, e per i miei pari, la superiorità di Riccardo Wagner sta appunto in questo: che la sua musica è, in gran parte bellissima, ed ha un alto e puro valore di arte indipendentemente dalla faticosa macchinazione teatrale e dalla significazione simbolica sovrapposta. [...] Come il lettore vede, non si tratta soltanto di un caso Wagner ma ben anche d'un caso Nietzsche. C'è qualcosa di frenetico in questo bizzarro libello: nella successione disordinata delle idee, nella incoerenza sintattica delle frasi, nella furia dell'invettiva. E pur tuttavia vi sono frequentissimi i bei lampi di verità e di ardire; e, certo, alcuni tra i principali caratteri della decadenza vi son descritti con sicura precisione. [...] Accuse, rampogne, ironie di tal genere sono ormai vanissime e indegne, specialmente d'un filosofo, anche se il filosofo «s'è messo fuori del suo tempo» ${ }^{25}$.

Torniamo a parlare dei romanzi di D'Annunzio. La presenza del Maestro tedesco che avvicina i romanzi Il trionfo della morte (1894) e Il fuoco (1900) induce

23 Ibidem, p. 65.

24 NIETZSCHE F. (2007), Il caso Wagner..., p. 66.

25 D'ANNUNZIO G., Il caso Wagner..., pp. 72 e 74. 
a parlare, per la durata di quasi un decennio, dal 1892 al 1900 con esattezza, di wagnerismo dannunziano. Tale periodo è però caratterizzato da due fasi nettamente distinte che la Corazzol fa corrispondere, più precisamente, alle date di stesura dei romanzi suddetti ${ }^{26}$ :

- I fase: dal 1892 al 1894, stesura finale del Trionfo

- II fase: dal 1894 al 1900, stesura finale del Fuoco

Specificatamente alla fase del Trionfo, nonostante i frequentissimi riferimenti musicali ad altri compositori come Chopin e Mendelssohn, risalta la trasposizione per intero del Tristano di cui si è già detto. L'opera scritta da D'Annunzio è un romanzo musicale, non c'è dubbio, esattamente come nelle intenzioni proclamate nella dedica al Michetti, ma non solo. Citare il superomismo significa, non di meno, ricondursi alla filosofia nietzscheana e farsene, forse meno esplicitamente e in misura meno dominante rispetto al pensiero wagneriano, diretto portavoce. L'ambientazione romana non deve dunque disorientare nel riconoscere lo spirito esclusivamente "germanico" che anima il pensiero e lo stile dello scrittore. I confini tra i concetti assoluti di Bene e di Male si fanno labili e poliedrici. In una «lotta breve e feroce» tra opposti sentimenti la morte irrompe come l'unica possibilità di rinascita e di riscatto da una vita inerte e improduttiva (due scene di suicidio aprono e chiudono la narrazione); l'odio è il motore, lo stimolo di tutte le vicende umane, di tutte le passioni e le emozioni, compreso l'amore. Parafrasando forse il Nietzsche di Al di là del bene e del male (1886) - ragion per cui occorrerebbe limitare i giudizi espressi dalla Sorge - l'intellettuale, seppur decadente e lontano da qualsiasi vitalismo superomistico, si ostina ancora a non arrendersi alla passiva accettazione della morale comune e fa della morte il suo «preludio di una filosofia dell' avvenire» ${ }^{27}$.

Con Il Fuoco (1900) si assiste, invece, ad un radicale cambio di direzione. Proprio negli anni immediatamente successivi alla pubblicazione del Trionfo, D’Annunzio inizia a maturare un forte interesse per il teatro. La città di Venezia,

${ }^{26}$ CFR. GUARNIERI CORAZZOL A. (1990), Il wagnerismo nei romanzi di D'Annunzio. Dalla prosa sinfonica al «pasticcio», in Sensualità senza carne..., pp. 147-171: "Nel D'Annunzio romanziere si può parlare di wagnerismo sistematico solo a partire dal 1892 e non oltre il 1900. [...] Esso si dichiarerà in due fasi ben distinte; culminando la prima nella versione finale del Trionfo, la seconda nella stesura, altrettanto dilazionata nel tempo, del Fuoco".

27 Così si conclude il romanzo: "Fu una lotta breve e feroce come tra nemici implacabili che avessero covato fino a quell'ora nel profondo dell'anima un odio supremo. E precipitarono nella morte avvinti", Trionfo..., p. 382. 
visitata per la prima volta nel 1887, appare, agli occhi dello scrittore, uno scenario teatrale perfetto, un vero e proprio palcoscenico urbano ${ }^{28}$. La città che forse più di tutte, forse ancor più di Roma, incarna l'arte, la bellezza e la storia, diventa così per D'Annunzio, il luogo più adatto in cui ambientare l'azione del romanzo. «A Venezia - dice Stelio Effrena - è impossibile sentire in modi diversi dai musicali [...] tutti i rumori vi si trasformano in voci espressive».

Se da una parte il Trionfo, con la trasposizione letteraria del Tristano, voleva apparire come una rivisitazione del pensiero wagneriano, nel Fuoco Wagner, pur assunto a protagonista della vicenda, passa gradualmente in sordina. Al sommo genio tedesco, simbolo per eccellenza del mondo germanico, si contrappone l'altrettanta genialità di un musicista italiano del Settecento. La città di Venezia, attraverso gli splendidi monumenti che l'hanno resa famosa e gli illustri artisti che ne hanno consacrato lo splendore, conserva in se stessa tracce memorabili della storia. Insieme a Claudio Monteverdi, Benedetto Marcello diventa il nuovo genio musicale italiano da emulare e la sua Arianna fa da sottofondo sonoro al romanzo. L'emulazione wagneriana diventa così agonismo: il germanesimo, precedentemente assunto a modello culturale, lascia il posto alla classicità italiana e latina rivendicata con orgoglio; l' «arte novella o rinnovellata» proclamata da Effrena, toltasi di dosso le barbare vesti e rifiutati i titanici spiriti combattenti, diventa quella squisitamente italiana. Wagner d'altronde è destinato a morire proprio a Venezia, a cadere «vinto» per lasciare spazio a un rinnovato, seppur antico, ideale di arte:

Io annunzio l'avvento di un'arte novella o rinnovellata che per la semplicità forte e sincera delle sue linee, per la sua grazia vigorosa, per l'ardore de' suoi spiriti, per la pura potenza delle sue armonie, continui e coroni l'immenso edifizio ideale della nostra stirpe eletta. Io mi orgoglio d'esser un latino [...] «Basta, basta!» gridò

${ }^{28} \mathrm{Si}$ legga, a tal proposito, il saggio di Giovanni Isgrò, Il Fuoco come laboratorio del teatro fuori dal teatro: "Venezia come set di se stessa, ma anche come impianto emblematico per una lettura totale ed en plein air del teatro festivo urbano. Un potenziale teatrico inteso come riscontro aperto a quell'utopia che solo la dimensione del romanzo può soddisfare. [...] tutta l'articolazione del set, lungi dal rimanere apparato scenografico, puro gioco dell'effimero, entra nello sviluppo dell'azione, partecipando (insieme agli effetti sonori, alle voci, al movimento) all'orchestrazione complessiva dell'evento descritto e idealmente rappresentato. [...] La dimensione totale del set urbano e l'idea del teatro/festa che ne consegue, spingono l'artista fino all'immaginazione di un dispositivo en plein air che non può non configurare inserito e perfettamente integrato nel tessuto monumentale della città e in particolare della città capitale, come contrapposizione massima della civiltà latina rispetto a quella 'barbara' e 'germanica' di Wagner'. Isgrò G. (1993), D’Annunzio e la mise en scène, Palumbo, Palermo, pp. 22 e 25. 
il pricipe Hodiz ridendo. «Il barbaro è vinto». «Non basta ancora» disse Antimo della Bella. «Bisogna glorificare il più grande degli innovatori, che la passione $\mathrm{e}$ la morte consacrarono veneziano, colui che ha il sepolcro nella chiesa dei Frari, degno d'un pellegrinaggio: il divino Claudio Monteverde». «Ecco un'anima eroica di pura essenza italiana!» assentì Daniele Glauro con reverenza. [... ] La mia opera è d'invenzione totale. Io non verso la mia sostanza in impronte ereditate. Io non debbo e non voglio obbedire se non al mio istinto, al genio della mia stirpe ${ }^{29}$.

Inoltre è certamente poco veritiera l'amicizia con Wagner vantata da D'Annunzio che, nei panni del suo alter ego Stelio, proprio alla fine del romanzo trasporta, insieme ad altri cinque giovani italiani, la bara del Maestro ${ }^{30}$. L'azione si svolge nel 1883, anno della morte di Wagner nella città lagunare ma, come dimostrato da Francesco Colombo in un articolo apparso sul «Corriere della Sera» il 17 marzo $1995^{31}$ e basandoci pure sulla Vita segreta di D'Annunzio (1938), attendibile biografia scritta dal suo segretario Tom Antongini, il primo viaggio compiuto dallo scrittore a Venezia avvenne nel 1887, quattro anni dopo la morte di Wagner.

Se nel Fuoco Wagner compare dunque in prima persona, egli appare in sordina, personaggio malato e morto nella conclusione dell'opera. Ed è curioso notare come sia proprio il secondo capitolo intitolato L'impero del silenzio ad avere, indipendentemente dalla presenza del Maestro tedesco, la più marcata musicalità. Confutare Wagner si traduce, in un certo qual senso, nel rinunciare alla coralità dei suoni e delle voci della sua musica. La vibrante polifonia dell'orchestra wagneriana cede così il passo alla musica del «silenzio» evocata da Daniele Glauro:

«è vero, Daniele, quel che tu mi comunicasti un giorno: la voce delle cose è essenzialmente diversa dal loro suono». [...] «E hai tu mai pensato che l'essenza della musica non è nei suoni?» domandò il dottor mistico. «Essa è nel silenzio che precede i suoni e nel silenzio che li segue. Il ritmo appare e vive in questi intervalli di silenzio. Ogni suono e ogni accordo svegliano nel silenzio che li precede e che li segue una voce che non può essere udita se non dal nostro spirito. Il ritmo è il

29 D’ANNUNZIO G. (1995), Il fuoco, Bit edizioni, Milano, pp. 72-73, 201.

30 Un attendibile e suggestivo resoconto sul soggiorno a Venezia di Wagner è stato fatto da Henry Perl che riferisce anche i nomi dei possibili traghettatori del feretro del Maestro. Cfr. perl H. (2000), Richard Wagner a Venezia, Marsilio, Venezia, p. 85 e p. 254 (nota n. 90).

31 COLOMBO F., D’Annunzio portò la bara di Wagner? Con la fantasia, "Corriere della Sera", 17 marzo 1995. 
cuore della musica, ma i suoi battiti non sono uditi se non durante la pausa dei suoni» ${ }^{32}$.

Il proclamato antiwagnerismo del Fuoco approda, con la prosa autobiografica del Notturno (1921), ad una ulteriore suggestione. Wagner, ormai morto, sembra essere fuori scena ma non del tutto. Se il Trionfo si configurava come una trasposizione narrativa dell'opera del Maestro tedesco, il Notturno prende spunto dall'opera lirica, questa volta non per riprodurla ma, addirittura, per rovesciarla. La trama del Tristano viene così, non esplicitamente ma significativamente, invertita. Eccone un suggestivo esempio:

Toglietemi da questa ambascia. Non resisto più. Scioglietemi da questo terrore. Non posso più respirare. Datemi un poco di luce. Aprite le finestre. Levatemi da questo buio spaventoso, dove non ho mai pace. Interrompete almeno per un'ora questo supplizio delle visioni, questo maritrio delle apparizioni orrende. Non so più resistere. Ho voglia di strapparmi le bende e di strapparmi gli occhi. Voi mi bendate la fronte, mi fasciate le palpebre, mi lasciate nell'oscurità. E io vedo, vedo, sempre vedo. $\mathrm{E}$ di giorno e di notte, sempre vedo ${ }^{33}$.

D'Annunzio, Notturno
Maledetto giorno con la tua luce! Veglierai tu sempre per mio tormento? La luce - quando si spegnerà? Quando si farà notte in questa casa? [...] Letizia senza misura, gioioso delirio! Nei vincoli del giaciglio come sopportarli? Orsù, in piedi, dove battono i cuori! Tristano, l'eroe, con la forza della gioia, si è dalla morte su svincolato! [...] (Si strappa le bende dalla ferita) Evviva, il mio sangue! Ch'esso scorra in letizia! (Balza dal suo giaciglio e s'avanza barcollando) Wagner, Tristano e Isotta

La prosa notturna e diaristica di D'Annunzio, ricca di immagini e di forme espressionistiche, raffigura un ridimensionato eroe assalito dalla malinconia e dal dolore. Come si può notare, leggendo l'esempio riportato, nel testo i suoni lasciano spazio alle visioni, alle immagini; la componente visiva domina su quella uditiva (si faccia attenzione alla ossessiva reiterazione del verbo «vedo»). Come già anticipato dalle parole di Glauro nel Fuoco, il silenzio diventa l'elemento sonoro predominante. E non è da sottovalutare come la scelta di un titolo spiccatamente melodico (il Notturno è un brano musicale in uso a partire dal XVIII secolo) abbia, in questo caso specifico, nulla a che vedere con la composizione

32 D'ANNUNZIO G. (1995), Il fuoco..., p. 117.

33 D'ANNUNZIO G. (1921), Notturno, Treves, Milano, p. 170. 
musicale. Nonostante queste considerazioni, anche nel racconto dei suoi mesi di cecità e di forzata inettitudine si intravede, come già notato da Alfredo Gargiulo sulla «Ronda» ${ }^{34}$, un D'Annunzio poeticamente musicista che, ascoltando questa volta i battiti del proprio cuore nel buio dell'oscurità e nell'immobilità dell'azione, pur percepisce dolci melodie silenziose:

Non mi sono mai sentito tanto pieno di musica come nelle pause della battaglia. [...] Le ore passano. La musica è come il sogno del silenzio. [...] E il cuore mi batte nel timore che un rintocco interrompa questa tacita musica ${ }^{35}$.

Si è così dimostrato come la musica abbia un ruolo di netta rilevanza all'interno della scrittura di D'Annunzio. Essa non è solo abbellimento estetico, sonora edulcorazione della sintassi, accompagnamento melodico alle vicende descritte. Si propone, piuttosto, come una vera e propria fonte di ispirazione, trama compositiva che motiva e supporta vicende e personaggi.

Analizzando i romanzi più marcatamente musicali, simbolo di opposte tendenze culturali e cambi di direzione concettuali, si è visto inoltre come la controversa figura di Wagner, la cui dottrina musicale ispira e condiziona l'intera trama narrativa del Trionfo, già nella prosa immediatamente successiva del Fuoco, in nome di un radicale rinnovamento della musica, ceda il passo alla musicalità del barocco italiano. La musica antica si offre, paradossalmente, come tramite per un radicale rinnovamento: si passa così dall'ideale «prosa moderna» del Trionfo, all'altrettanto ideale «musica moderna» di impronta squisitamente italiana.

34 Gargiulo, oltre a notare nel Notturno un notevole cambiamento dello stile dannunziano, evidenziando una «particolare immediatezza e semplicità stilistica» e un «abbassamento del chiuso e imperativo io», in merito alla musicalità del romanzo scrive: «Così, assai probabilmente, nella sua intenzione il Notturno dovette svolgersi, sì, come racconto più o meno realistico di quelle vicende, ma anche come una specie di composizione musicale, un seguito tutto legato di motivi. [...] Fu illusione: anche se alla parola 'musica' si attribuisce il senso traslato, che solo le conviene allorché si tratta di poesia. A meno che si vogliano chiamar 'musicali' soprattutto i passaggi, rapidi o graduali, dai momenti di pena a quelli di sollievo; poiché tanto e non più la preoccupazione musicale del poeta mi pare riesce a ottenere. [...] Sarebbe da osservare, piuttosto dove la musica ha nuociuto senz'altro agli elementi e rapporti della figurazione poetica. Le divagazioni, i corpi estranei (uno fra tutti: il pezzo sui violoncelli), hanno un'origine puramente musicale. [...] Qualcosa che qui si distingue, fece in realtà tutt'uno con la musicalità, nei propositi strutturali; e cioè la tendenza impressionistica», "La Ronda" XI-XII, 1921, pp. 746-772.

35 D’ANNUNZIO G. (1921), Notturno, Treves, Milano, pp. 216, 243, 481. 
A far da sottofondo, invece, alla scrittura intimistica del Notturno è la «musica del silenzio» grazie alla quale, dietro le immagini dei ricordi, i turbinii dei pensieri e la materialità delle cose, è comunque sempre possibile cogliere echi sonori, voci melodiose, rimbombi di una roboante sinfonia wagneriana. Il Maestro tedesco infatti, seppure impercettibilmente, continua a sopravvivere e a farsi, ma per antitesi, ancora una volta ispiratore tout court della prosa dannunziana.

\section{Summary \\ "YOU WON THE BARBARIAN". \\ D'ANNUNZIO CONTRA WAGNER. \\ SYMPHONIC FROM GERMAN TO MUSIC OF SILENCE IN THE NOVELS OF GABRIELE D'ANNUNZIO}

This study aims to demonstrate how music has a relevance in the prose of Gabriele D'Annunzio. It is not just an aesthetic embellishment, sound sweetening syntax, melodic accompaniment to the events described. It suggests, rather, as a real source of inspiration, texture composition that motivates and supports events and characters. By analyzing the novels most markedly musical, symbol of opposing cultural trends and conceptual changes of direction, is possible to see as a controversial figure of Wagner, whose music inspires and influences the novel Il trionfo della morte, already in the prose immediately following, Il fuoco, in the name of a radical renewal of the music gives way to the musicality of the Italian Baroque. Ancient music is offered, paradoxically, as a vehicle for a radical renewal: from the ideal german "modern prose" of Trionfo, D'Annunzio arrives to equally ideal prose inspired by italian "modern music".

\section{Streszczenie}

Niniejszy esej podkreśla rolę muzyki w prozie Gabriela D’Annunzia. Nie chodzi tylko o funkcję estetyczną dzieła literackiego, ale o uporządkowaną dźwiękowo polifoniczną warstwę, która stanowi koherentną całość; sama muzyka winna towarzyszyć opisywanym wydarzeniom i postaciom w jego utworach. Autor podkreśla, że głównie w muzyce możemy znaleźć prawdziwe źródło inspiracji; ma ona wpływ na strukturę kompozycyjną dzieła i stanowi nieodłączny element wielu wydarzeń; muzyka podkreśla także egzystencjalny aspekt życia protagonistów. Przeprowadzone analizy wybranych nowel są wyrazem sprzeciwu wobec tendencji panujących w kulturze europejskiej; ten fenomen obserwujemy na przykładzie muzyki Wagnera. Wagnerowska muzyka wywiera wpływ na tematykę i strukturę nowel, takich jak: Triumf śmierci i Ogień. D’Annunzio w imię swojej 
radykalnej odnowy opiera się na muzyce włoskiego baroku. Jednak muzyka dawna pojawia się u D’Annunzia jako sposób na dokonanie zmian, które oscylują pomiędzy idealistyczną ,,nowoczesną prozą" niemiecką (Triumf) a idealistyczną prozą inspirowaną przez „nowoczesną muzykę” włoską.

Słowa klucze: muzyka, Modern Romance, Gabriele D’Annunzio, Richard Wagner, Friedrich Nietzsche. 